\title{
Thromboprophylaxis use in medical and surgical inpatients and the impact of an electronic risk assessment tool as part of a multi-factorial intervention. A report on behalf of the elVis study investigators
}

\author{
Edward Janus • Anmol Bassi · David Jackson • \\ Harshal Nandurkar $\cdot$ Mark Yates
}

Published online: 4 June 2011

(c) The Author(s) 2011. This article is published with open access at Springerlink.com

\begin{abstract}
Venous thromboembolism (VTE) is a major source of morbidity and mortality for both surgical and medical hospitalised patients. Despite the availability of guidelines, thromboprophylaxis continues to be underutilised. This study aims to assess the effectiveness of an electronic VTE risk assessment tool (elVis) on VTE prophylaxis in hospitalised patients. A national, multicentre, prospective clinical audit collected information on VTE prophylaxis and risk factors for VTE in 2,400 hospitalised patients (comprising of equal numbers of medical, surgical and orthopaedic patients). After auditing the standard care use of VTE prophylaxis in 1,200 consecutive patients (audit 1 , A1), the elVis system was installed and a second audit (A2) of VTE prophylaxis was performed in a further 1,200 patients. The use of the electronic VTE risk assessment tool was low with $20.5 \%$ of patients assessed with elVis. The intervention, elVis plus accompanying education, improved
\end{abstract}

E. Janus $(\bowtie)$

Western Hospital, 469 Great Western Highway, Pendle Hill, NSW 2145, Australia

e-mail: edwarddj@unimelb.edu.au

\section{A. Bassi}

Frankston Hospital, Hastings Road, Frankston, VIC 3199,

Australia

D. Jackson

Lismore Base Hospital, 70 Hunter Street, Lismore, NSW 2480,

Australia

H. Nandurkar

St Vincent's Hospital, 41 Victoria Parade, Fitzroy, VIC 3065, Australia

M. Yates

Ballarat Health Services, Drummond Street, Ballarat, VIC 3350,

Australia the use VTE prophylaxis to guidelines by 5.0\% amongst all patients and by $10.7 \%$ amongst high risk patients (adjusted odds ratio (AOR) 1.27 and 1.65 respectively). The use of elVis in A2 varied between hospitals and specialties and this resulted in marked heterogeneity. Despite this heterogeneity, patients assessed with elVis had 1.44 times higher AOR of being treated to guidelines compared to those who were not $(P<0.05)$. The use of elVis accompanied by staff education improved VTE prophylaxis, especially amongst high risk patients. To optimise the effectiveness and support enduring practice change electronic systems, such as elVis, need to be completely integrated within the treatment pathway.

Keywords Deep vein thrombosis · Venous thromboembolism · Pulmonary embolism · Guideline adherence $\cdot$ Prevention $\cdot$ Thromboprophylaxis

\section{Introduction}

Venous thromboembolism (VTE), which comprises of pulmonary embolism (PE) and deep vein thrombosis (DVT), is a major source of morbidity and mortality for both surgical and medical hospitalised patients. It is the most common preventable cause of hospital-related death [1], yet despite the availability of clinical guidelines in Australia [2, 3] and internationally [4, 5], thromboprophylaxis continues to be underutilised $[1,6]$ and has been identified as "the number one strategy to improve patient safety in hospitals" [5].

In 2008, it is estimated that there were over 14,500 cases of VTE and approximately 5,000 deaths due to VTE in Australia. The estimated financial cost of VTE was in excess of AU $\$ 1.7$ billion, with $80 \%$ of these costs due to 
lost productivity as a result of premature death [7]. Western Australian hospital morbidity data determined that in 1999-2001 the rate of VTE was 80 per 100,000 hospitalised patients with equal proportions arising from medical and surgical admissions. Over half of secondary cases of VTE occurred as readmissions within 3 months of the original hospital admission [8]. Most hospitalised patients have one or more risk factors for VTE [3]. Surgery is a well established VTE risk factor and the use of thromboprophylaxis is generally higher amongst surgical patients than medically ill patients [9], however VTE cases in acute hospital settings are equally attributable to medical and surgical admissions [10]. In addition, 50-70\% of symptomatic events and $70-80 \%$ of fatal PEs occur in nonsurgical patients [5, 11-14].

The ENDORSE study has demonstrated that VTE prophylaxis is suboptimal and that the use of thromboprophylaxis in medically ill patients has particular scope for improvement [9]. Amongst medically ill patients, prophylaxis appears to correlate with disease severity rather than medical diagnosis, for example the recommended prophylaxis was more likely to be instituted if the patient was admitted into intensive care or required central venous catheters, markers of severe disease [15].

There have been numerous efforts made to improve VTE prophylaxis. Systematic reviews indicate that passive methods, such as the dissemination of guidelines, is unlikely to translate into improved practice [16-20]. Factors that appear to improve VTE prophylaxis are systems that remind clinicians to assess the VTE risk status of patients, and then assist clinicians prescribe the appropriate prophylaxis for the risk classification. Studies which have used electronic systems to facilitate these processes appear promising in improving VTE prophylaxis [1, 21].

We report the results of a multicentre clinical audit before and after an intervention, examining the effectiveness of an electronic risk assessment system on VTE prophylaxis in hospitalised patients. The primary objective was to determine the effect on the rate of VTE prophylaxis in hospitalised patients of an electronic risk assessment tool that guides appropriate management according to guidelines or local protocols. Secondary objectives were to assess the appropriateness of VTE prophylaxis in hospitalised medical, surgical and orthopaedic patients and to describe the types of VTE prophylaxis prescribed.

\section{Methods}

A multicentre prospective audit was performed in 6 hospitals across Australia. The audit collected information on VTE prophylaxis and risk factors for VTE in 2,400 hospitalised patients. The selection and use of VTE prophylaxis was based on standard care and was not determined by the audit protocol.

Participants in the audit were adult patients (aged $\geq 18$ years) hospitalised for at least $24 \mathrm{~h}$ in a medical or surgical ward. Patients were excluded from the study if they had participated in a VTE study in the last 90 days, or if they were admitted to the following hospital wards; intensive care, coronary care, paediatrics, maternity, gynaecology, or psychiatry. Individual patients could only be audited once, if they were re-admitted to hospital, VTE prophylaxis was not audited on subsequent admissions.

Each study site performed a baseline audit of 120 or 240 consecutive patients (comprising of equal numbers of medical, surgical and orthopaedic patients) regarding VTE risks, prophylaxis and bleeding risks. This data was recorded on a data collection form, to reflect the conditions present on admission or developed whilst in hospital, regardless of the length of hospital stay. After completing the first audit (A1), the electronic VTE risk assessment system was installed by Core Medical Solutions into each participating hospital. The electronic VTE risk assessment system allowed VTE risk to be classified as high or not high for each patient, based on local hospital guidelines, and the system flagged any patient that had not had their VTE risk assessed. Implementation of the electronic system was accompanied by medical officer education on its use and a general increase in awareness of VTE prophylaxis. After the electronic risk assessment tool was embedded within the hospital, a second audit (A2) of VTE risks and prophylaxis was conducted on an additional 120 or 240 consecutive patients. The second audit was completed as early as 5 months and no later than 10 months after the first audit. The number of patients to be audited at each hospital was based on the hospital's capacity to complete the audit within the study timeframe. Four hospitals recruited 240 patients in each audit cycle and two hospitals recruited 120 patients per cycle.

This audit was conducted in accordance with the Declaration of Helsinki [22] and written approval was obtained from the relevant Human Research Ethics Committee at each study site. Patient consent was not obtained as the process of obtaining consent posed significant risk of influencing physician behaviour. This was deemed acceptable, as selection of VTE prophylaxis was based on standard care and not by the audit protocol.

We estimated that a total of 2,400 evaluable patients would be required in the audit (1200, 400 medical, 400 surgical and 400 orthopaedic, in each audit cycle) to detect a difference between an A1 (baseline) proportion of patients treated to guidelines of 0.60 and an A2 proportion of patients treated to guidelines of 0.70 (odds ratio $=1.556)$ and to have a power of at least $80 \%$ (5\%, two-sided significance level). Data were summarised using descriptive 
statistics. The normal approximation method was used to calculate $95 \%$ confidence intervals for all sites combined. Logistic regression was used to evaluate the primary end point as to whether the use of the electronic risk assessment system improved the appropriate use of thromboprophylaxis at A2 versus A1 (standard care). Odds ratios for the effect of the electronic risk assessment tool were adjusted for the study design (site and patient specialty type) and for risk status (high risk, not high risk), as appropriate. All statistical analyses were performed using SAS $^{\circledR}$ Version 9.2 .

\section{Results}

A total of 2,406 patients were audited in this study, 1,206 in Audit 1 (A1), 402 medical, 404 surgical and 400 orthopaedic and 1,200 patients in Audit 2 (A2), 401 medical, 398 surgical and 401 orthopaedic. Demographic data for the patients audited are summarised in Table 1. Patients were well matched for all demographic measures between the two audits, as well as within each patient specialty type. In A1, 947 (78.5\%) patients were high risk at admission and a similar percentage $78.7 \%$ (944) were high risk in A2. As expected, high risk patients were older (mean age 67.8 years, A1 and 66.0 years, A2) compared to not high risk patients (mean ages 49.1 years, A1 and 54.5 years, A2).

The VTE risk factors found in patients are shown in Table 2. Orthopaedic patients generally had fewer risk factors however there was a considerable difference in A1 compared to A2 (no risk factors in $76.5 \%$ vs. $52.4 \%$, $P<0.05)$. Differences were also observed in the prevalence of specific co-morbidities that increased VTE risk between the two audits, for example active cancer was more prevalent in A1 than A2, especially amongst medical patients, while acute inflammation was more prevalent in A2 than A1 amongst surgical and orthopaedic patients. The number of VTE risk factors per patient is illustrated in Fig. 1. The most common VTE risk factors reported in A1 were acute infection or acute inflammatory disorder $(31.7 \%, 382 / 1206)$ and active cancer $(10.8 \%, 130 / 1206)$. These risk factors were also the most common in A2 (Table 2). In both audits, only a minority of patients had a risk factor for bleeding at admission (A1, 9.5\% 114/1206 and A2, 5.8\% 69/1200). The most common bleeding risk was hepatic impairment (Table 2). Almost $20 \%$ of patients had a contraindication to adding thromboprophylaxis in the audit (A1, 19.0\% 229/1206, and A2, 16.0\% 192/1200). The most common contraindications for initiating thromboprophylaxis included high risk of bleeding, active bleeding and range of other contraindications such as, renal impairment, chronic kidney disease, palliative care and falls risk. Seventy-two patients in A1 and 60 patients in A2 were already taking thromboprophylaxis at admission (Table 2).

In A1, 66.8\% (806/1206) of all patients and 63.5\% (601/ 947) of high risk patients were treated to guidelines. This increased by $5.0 \%$ to $71.8 \%(862 / 1200)$ of all patients $(P<0.05)$ and by $10.7 \%$ to $74.2 \%(700 / 944)$ for high risk patients $(P<0.05)$ (Fig. 2a, b). The adjusted odds ratio (AOR) of being treated to guidelines as a result of participating in the audit increased significantly by 1.27 (95\% CI 1.07-1.49), indicating that patients in A2 had a 1.27 times higher odds, adjusted for risk status, of receiving appropriate VTE prophylaxis than from standard care, in A1. Similarly the AORs of being treated to guidelines for high risk patients was 1.65 (95\% CI 1.37-1.99, $P<0.05$ ).

The use of the electronic risk assessment tool in A2 to assess VTE risk varied between the participating centres, ranging from none to half of the patients assessed using this tool. Overall elVis was used in $22.6 \%$ (213/944) of high

Table 1 Patient demographics

\begin{tabular}{|c|c|c|c|c|c|c|c|c|}
\hline & \multicolumn{2}{|l|}{ Medical } & \multicolumn{2}{|l|}{ Surgical } & \multicolumn{2}{|l|}{ Orthopaedic } & \multicolumn{2}{|l|}{ Total } \\
\hline & $\begin{array}{l}\text { Audit } 1 \\
(\mathrm{n}=402)\end{array}$ & $\begin{array}{l}\text { Audit } 2 \\
(\mathrm{n}=401)\end{array}$ & $\begin{array}{l}\text { Audit } 1 \\
(\mathrm{n}=404)\end{array}$ & $\begin{array}{l}\text { Audit } 2 \\
(\mathrm{n}=398)\end{array}$ & $\begin{array}{l}\text { Audit } 1 \\
(\mathrm{n}=400)\end{array}$ & $\begin{array}{l}\text { Audit } 2 \\
(\mathrm{n}=401)\end{array}$ & $\begin{array}{l}\text { Audit } 1 \\
(\mathrm{n}=1206)\end{array}$ & $\begin{array}{l}\text { Audit } 2 \\
(\mathrm{n}=1200)\end{array}$ \\
\hline Male $(\%)$ & $213(53.0 \%)$ & $198(49.4 \%)$ & $225(55.7 \%)$ & $220(55.3 \%)$ & $183(45.8 \%)$ & $191(47.6 \%)$ & $621(51.5 \%)$ & $609(50.8 \%)$ \\
\hline Female (\%) & $189(47.0 \%)$ & $203(50.6 \%)$ & $178(44.1 \%)$ & $178(44.7 \%)$ & $217(54.3 \%)$ & $210(52.4 \%)$ & $584(48.4 \%)$ & $591(49.3 \%)$ \\
\hline Mean age years (SD) & $71.1(17.7)$ & $73.7(16.2)$ & $58.2(20.2)$ & $56.6(20.3)$ & $62.1(19.4)$ & $60.2(19.7)$ & $63.8(19.9)$ & $63.5(20.2)$ \\
\hline Mean weight kg (SD) & $\begin{array}{l}71.5(21.4) \\
(\mathrm{n}=97)\end{array}$ & $\begin{array}{l}71.4(21.8) \\
(\mathrm{n}=121)\end{array}$ & $\begin{array}{l}78.9(19.8) \\
(\mathrm{n}=250)\end{array}$ & $\begin{array}{l}78.2(19.5) \\
(\mathrm{n}=219)\end{array}$ & $\begin{array}{l}82.7(19.7) \\
(\mathrm{n}=219)\end{array}$ & $\begin{array}{l}82.0(19.8) \\
(\mathrm{n}=238)\end{array}$ & $\begin{array}{l}79.1(20.4) \\
(\mathrm{n}=566)\end{array}$ & $\begin{array}{l}78.3(20.5) \\
(\mathrm{n}=578)\end{array}$ \\
\hline Mean height $\mathrm{cm}$ (SD) & $\begin{array}{l}169.0(10.0) \\
(\mathrm{n}=13)\end{array}$ & $\begin{array}{l}165.3(9.0) \\
(\mathrm{n}=15)\end{array}$ & $\begin{array}{l}167.6(11.2) \\
(\mathrm{n}=144)\end{array}$ & $\begin{array}{l}167.5(10.7) \\
(\mathrm{n}=130)\end{array}$ & $\begin{array}{l}167.0(11.3) \\
(\mathrm{n}=149)\end{array}$ & $\begin{array}{l}166.7(11.6) \\
(\mathrm{n}=193)\end{array}$ & $\begin{array}{l}167.3(11.2) \\
(\mathrm{n}=306)\end{array}$ & $\begin{array}{l}166.9(11.1) \\
(\mathrm{n}=338)\end{array}$ \\
\hline Mean BMI kg/m² (SD) & $\begin{array}{l}20.7(6.8) \\
(\mathrm{n}=10)\end{array}$ & $\begin{array}{l}22.1(5.6) \\
(\mathrm{n}=14)\end{array}$ & $\begin{array}{l}27.9(7.4) \\
(\mathrm{n}=142)\end{array}$ & $\begin{array}{l}27.7(5.7) \\
(\mathrm{n}=130)\end{array}$ & $\begin{array}{l}30.4(6.6) \\
(\mathrm{n}=145)\end{array}$ & $\begin{array}{l}30.2(7.9) \\
(\mathrm{n}=190)\end{array}$ & $\begin{array}{l}28.9(7.3) \\
(\mathrm{n}=297)\end{array}$ & $\begin{array}{l}28.9(7.3) \\
(\mathrm{n}=334)\end{array}$ \\
\hline
\end{tabular}




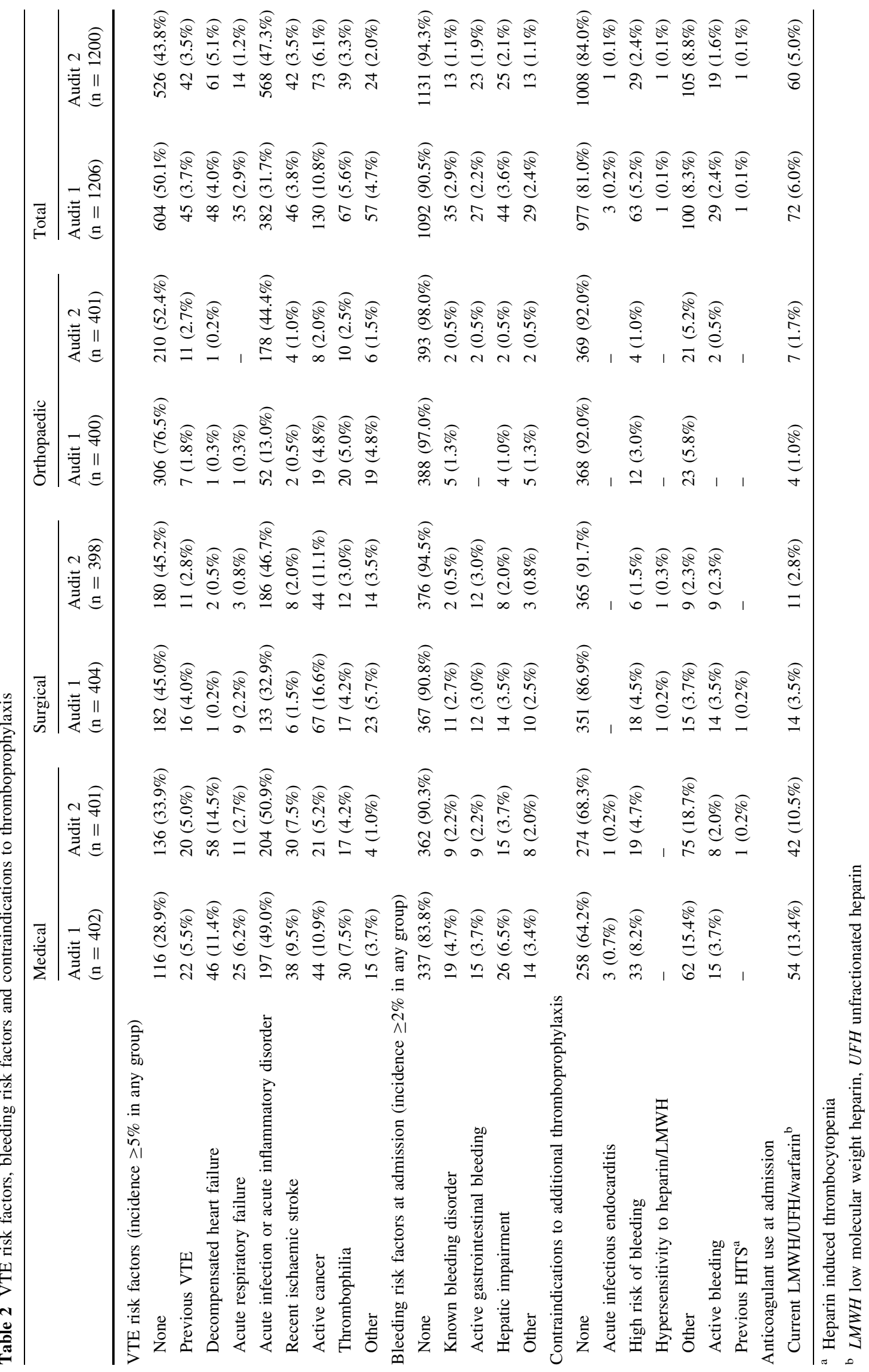


risk patients and $20.5 \%(246 / 1,200)$ of all patients. Its use also differed between specialty types, with highest use amongst medical patients $35.2 \%$ (141/401), followed by surgical patients, $17.8 \%$ (71/398) and orthopaedic patients,



Fig. 1 Number of VTE risk factors per patient (all patients)

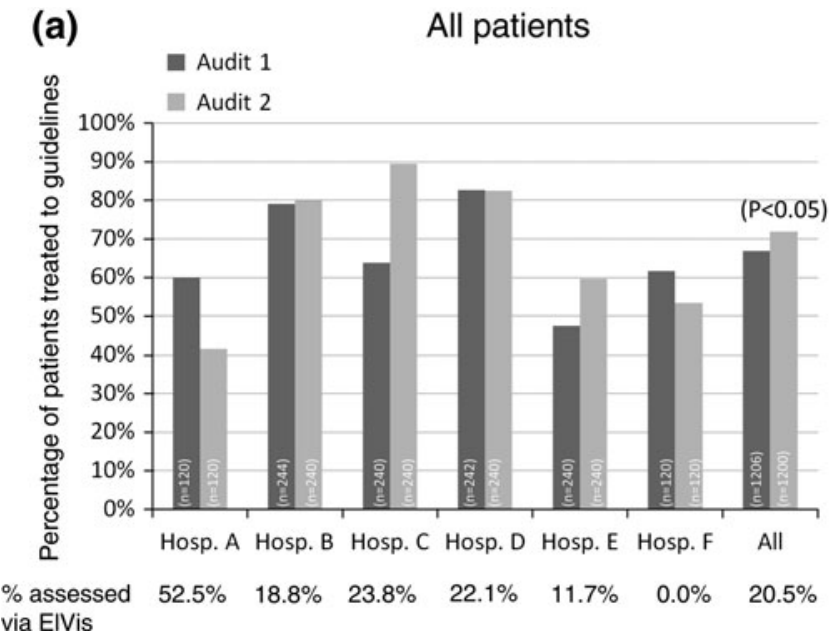

$8.5 \%(34 / 401)$. Due to the low use of the electronic risk assessment tool, an additional logistic regression analysis was conducted to determine its impact amongst patients in audit 2 only. Seventy-eight percent (192/246) of patients assessed using the electronic risk assessment tool received appropriate prophylaxis versus $70.2 \%$ (670/954) for those whose risk was assessed using another method. Adjusting for risk status, patients assessed with electronic risk assessment had 1.44 times greater odds of receiving appropriate VTE prophylaxis than patients who were not assessed using this system (AOR 1.44, 95\% CI 1.04, 1.99). Statistically significant improvements in the percentage of all patients treated to guidelines were observed in A2 for both medical patients and orthopaedic patients (AOR 1.56 and 1.36 respectively) but not for surgical patients (Fig. 3a). Similar findings were observed amongst high risk patients; however the study was not powered to detect significant differences in this subanalysis (Fig. 3b).

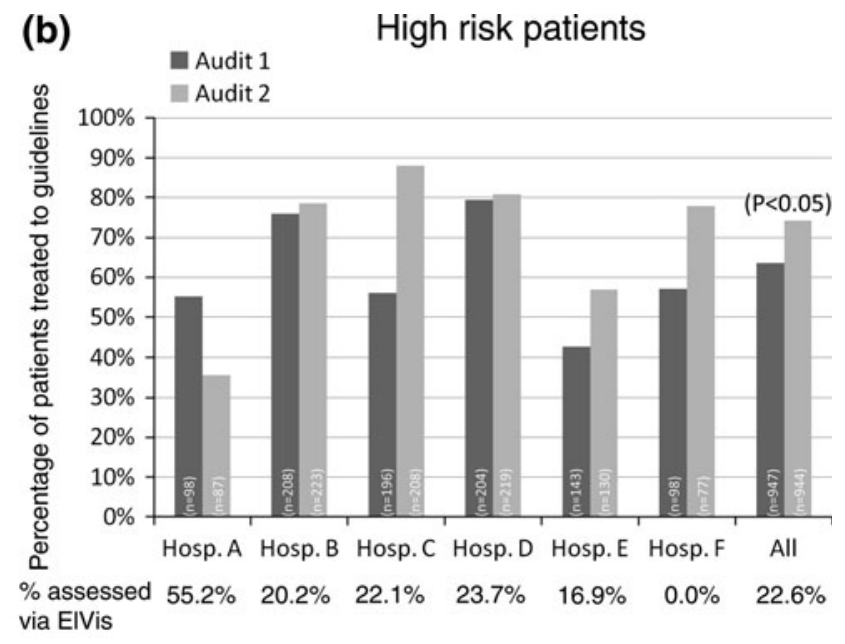

Fig. 2 Percentage of patients treated to guidelines. a All patients. b High risk patients


Fig. 3 Percentage of patients treated to guidelines by specialty group. a All patients. b High risk patients 


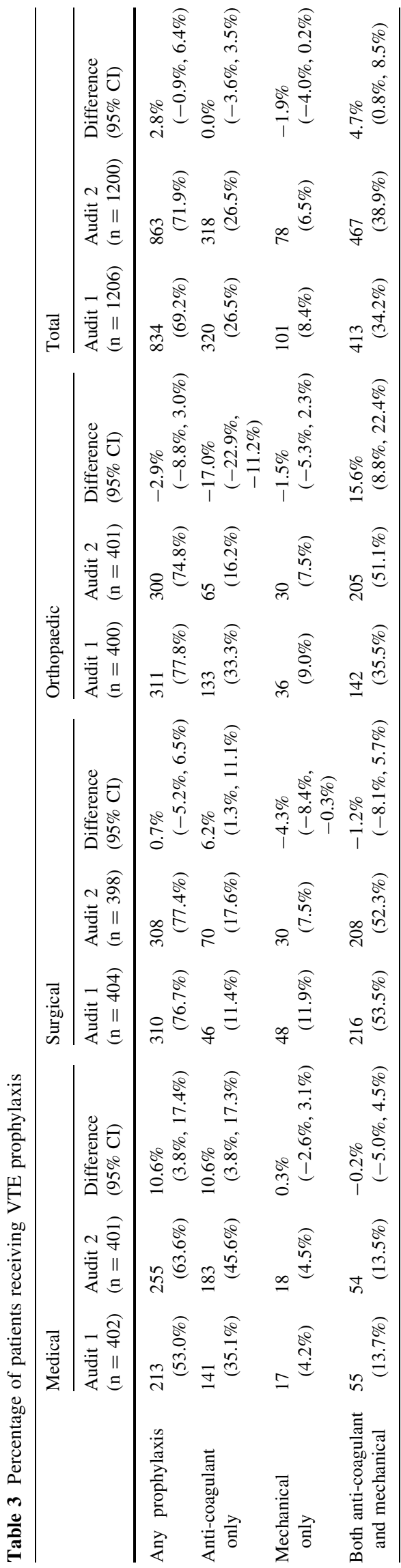

The types of VTE prophylaxis prescribed in both audits are shown in Table 3. The level of VTE risk and bleeding risk differed between $\mathrm{A} 1$ and $\mathrm{A} 2$ and this influenced the use of VTE prophylaxis. The percentage of patients overall receiving any VTE prophylaxis in A2 compared to baseline (A1) increased by $2.8 \%$ (not statistically significant), with a statistically significant increase observed for the use of the combination of anti-coagulant and mechanical prophylaxis.

The most frequently prescribed anticoagulant prophylaxis in all patients was enoxaparin $(42.9 \%$ in $\mathrm{A} 1$ and $46.4 \%$ in A2), followed by unfractionated heparin and dalteparin. The prescribing of enoxaparin increased in A2 amongst both medical and surgical patients, but decreased amongst orthopaedic patients. Unfractionated heparin prescribing decreased amongst surgical patients but increased amongst medical patients. Dalteparin was only used amongst orthopaedic patients. The most frequently prescribed mechanical prophylaxis in patients overall was graduated compression stockings.

\section{Discussion}

The routine use of VTE prophylaxis in hospitalised patients is generally suboptimal, with the percentage of at risk patients who receive prophylaxis according to guidelines ranging from 13 to $64 \%$, depending on the patient population being investigated [23-26]. Data from the multinational ENDORSE study demonstrated that less than half of patients at risk received prophylaxis and prophylaxis was generally higher amongst surgical patients than medical patients (58.5\% surgical and 39.5\% medical) [9]. The hospitals that participated in our study performed VTE prophylaxis at a relatively high rate in $\mathrm{A} 1$, prior to the intervention, with approximately two-thirds of both, all patients and high risk patients receiving appropriate prophylaxis in A1. Despite these high baseline levels implementing the electronic risk assessment tool and the accompanying education activities resulted in further improvements in VTE prophylaxis, increasing by $5.0 \%$ for all patients and $10.7 \%$ for patients at high risk. This finding is consistent with that of other investigators that confirm that implementing active strategies that remind clinicians to assess VTE risk and assist with appropriate prescribing are effective [21, 27, 28]. For example, Durieux et al. investigated the use of a computer-based clinical decision support system on VTE prophylaxis amongst orthopaedic patients. Use of the computerised system further improved the percentage of patients treated to guidelines by $12.1 \%$, from 82.8 to $94.9 \%$ [28].

Our study also investigated VTE prophylaxis amongst three discrete patient specialty types, medical, surgical and orthopaedic patients, in equal proportions. Previous studies 
have indicated that VTE prophylaxis is generally implemented better amongst surgical patients than medical patients [9]. Although a higher percentage of surgical patients received appropriate prophylaxis $(72.8 \%)$ in $\mathrm{A} 1$, the rate of prophylaxis according to guidelines amongst medical patients was also high at $67.4 \%$ and higher than orthopaedic patients at $60.3 \%$. Improvement in VTE prophylaxis was greatest amongst medical patients $(8.9 \%$ amongst all medical patients and $18.6 \%$ amongst high risk medical patients). Significant improvements were also observed amongst orthopaedic patients, whilst no significant change was observed amongst surgical patients. These differences between patient specialty type are partially explained by the differing use of the electronic risk assessment tool, which was most frequently used with medical patients.

There was marked heterogeneity between the audited hospitals and within patient specialty types. Hospitals and specialty subgroups with the highest percentage of appropriate prophylaxis use in $\mathrm{A} 1(>80 \%)$ generally demonstrated little change (marginal improvement) in VTE prophylaxis as a result of participation in the audit. Amongst the remaining hospitals three demonstrated improvements in VTE prophylaxis, whilst in one hospital the intervention appeared to impede appropriate prophylaxis.

The main limitation of our study resulted from the low and variable use of the electronic risk assessment tool, ranging from one hospital not assessing any patients with the tool to its use to assess two-thirds of all medical patients in another hospital. This variation arose from several factors of which the two most significant were software related issues and clinical leadership. In the pilot study conducted at Geelong Hospital, the electronic risk assessment tool was fully integrated within the hospital admission system [29]. Hence, the risk assessment tool formed a seamless part of the admission system. For all the hospitals in our study, elVis was not fully integrated within the hospital's patient admission system but was a separate, stand alone application. This created a major impediment to its routine use, as clinicians needed to specifically open the electronic risk assessment tool to assess VTE risk. In addition, the routine use of the electronic risk assessment system was further compromised by implementation issues that arose at some hospitals, where synchronisation of patient lists between the hospital admissions system and the VTE risk assessment tool was not always achieved with $100 \%$ accuracy. When patients were missing from the VTE database, VTE risk assessment defaulted to standard care. The importance of clinical leadership was clearly demonstrated from within hospital comparisons between patient specialty types. Where clinical leadership was strong and supportive of electronic risk assessment, usually in medical departments, so was the use of the tool. Although patients in each audit were well matched based on demographic variables, differences in risk factors were observed. Whilst adjustments have been made for measured confounders, it is not possible to account for unmeasured confounders and their potential impact on our results. Other limitations of our study are the multiple statistical analyses conducted increasing the chance of false positive results, the lack of clarity as to the effect of elVis over education and whether these improvements in VTE prophylaxis are sustainable.

Despite the varied use of elVis, analysis of patients within the second audit identified a clinical benefit associated with the use of this tool. Patients assessed using the electronic risk assessment had 1.44 times higher odds, adjusted for risk status, to receive appropriate VTE prophylaxis than patients who were not assessed using this system $(P<0.05)$.

The most significant challenge in the area of VTE prophylaxis is the application of existing evidence into everyday clinical practice. Quality improvement and practice change is possible but for it to be sustained it requires that the change be integrated into daily patient care [21, 30, 31]. The use of computerised risk assessment and reminder systems make this possible and has been demonstrated to be effective tools of change in the area of preventative care [27-30]. For example, a randomised clinical trial in 2,506 hospitalised patients demonstrated that the use of computerised alert system increased physician use of VTE prophylaxis versus control $(33.5 \%$ vs. $14.5 \%, P<0.001)$ and reduced the risk of DVT or PE at 90 days by $41 \%$ $(P=0.001)$ [27]. In addition, a systematic review by Tooher et al. [21], found that computer based clinical decision support systems were amongst the most effective strategies for improving prescribing practice as they minimise errors made by clinicians with varying degrees of knowledge, interest and motivation for VTE prevention. This review also identified that the studies that achieved at least $90 \%$ adherence to VTE guidelines involved either an iterative process of audit and review or an active reminder system.

In our study, the absolute improvement in the percentage of patients that were treated to guidelines was $5.0 \%$ amongst all patients and $10.7 \%$ amongst high risk patients as a result of the audit intervention. The audit intervention, although focused on the use of the electronic risk assessment tool was effectively a multimodal intervention, involving clinical leadership, professional education, increased awareness of VTE prophylaxis, as well as the use of the elVis electronic risk assessment tool. The absolute improvement in appropriate VTE prophylaxis increased by $7.8 \%$ amongst patients who were assessed using the electronic risk assessment compared to not using this tool. This level of absolute improvement in clinical practice is comparable to other change management tools aimed at 
improving guideline implementation. Grimshaw et al. [32] identified that reminder systems were the most effective tool for change (median absolute effect of $13.1 \%$ ), followed by healthcare professional education at $8.1 \%$ (noting that the benefits from this tool of change is short-lived), audit and feedback $7.0 \%$ and multifaceted interventions involving educational outreach at $6.0 \%$.

\section{Conclusion}

The use of elVis, an electronic VTE risk assessment tool, accompanied by staff education overall improved VTE prophylaxis, especially amongst high risk patients. However, the response to this intervention varied between participating hospitals and between medical, surgical and orthopaedic patients. Our study confirmed that the ongoing challenge of applying VTE prophylaxis guidelines into routine clinical practice can be assisted with the use of electronic assessment and decision support tools. To be maximally effective and to deliver enduring practice change, these tools need to be fully integrated within the treatment pathway in a readily accessible, easy to use manner.

Acknowledgments The elVis study was sponsored by sanofiaventis australia pty ltd. The authors would like to acknowledge the contribution of study nurse coordinators, Sofi Milenkovski, Carmel Goss, Su-Ann Hampson, Jenny Hiew, Jan deClifford, Bridget Ady, Janice Boys and all the clinicians that participated in this clinical audit. The authors would like to thank Ronelle Heath and OakPil Han of Novotech for assistance with statistical analyses and George Krassas of Scius Solutions Pty Ltd for assistance in the preparation of this manuscript; their contributions have been funded by sanofiaventis australia pty ltd.

Conflict of interest Professor Edward Janus: In addition to the funding of the research nurse for the 2010 elVis study I have received a further 2 grants over 2007-2009 for \$99,500 from sanofi-aventis to employ a research nurse to work on improving VTE prophylaxis. I received sanofi-aventis support to attend one Conference on VTE prophylaxis in Sydney. Dr Jackson, Dr Anmol Bassi, Professor Nandurkar and Associate Professor Mark Yates have no declared conflict.

Open Access This article is distributed under the terms of the Creative Commons Attribution Noncommercial License which permits any noncommercial use, distribution, and reproduction in any medium, provided the original author(s) and source are credited.

\section{References}

1. Michota FA (2007) Bridging the gap between evidence and practice in venous thromboembolism prophylaxis: the quality improvement process. J Gen Intern Med 22(12):1762-1770
2. Cardiovascular Expert Group (2008) Prevention of venous thromboembolism. In: Therapeutic guidelines: cardiovascular, ed Version 5. Therapeutic Guidelines Ltd, Melbourne

3. Australia and New Zealand Working Party on the Management and Prevention of Venous Thromboembolism (2007) Prevention of venous thromboembolism best practice guidelines for Australia and New Zealand, 4th edn. Health Education and Management Innovations, Sydney

4. Cardiovascular disease educational and research trust, Cyprus Cardiovascular Disease Educational and Research Trust, European Venous Forum, International Surgical Thrombosis Forum, International Union of Angiology, Unnion Internationale de Phlebologie (2006) Prevention and treatment of venous thromboembolism. Int Angiol 25:101-161

5. Geerts WH, Bergqvist D, Pineo GF, Heit JA, Samama CM, Lassen MR, Colwell MR (2008) Prevention of venous thromboembolism: American College of Chest Physicians Evidence-Based Clinical Practice Guidelines (8th edition). Chest 133(6 Suppl):381S453S

6. Gallagher M, Oliver K (2009) Hurwitz M: improving the use of venous thromboembolism prophylaxis in an Australian teaching hospital. Qual Saf Health Care 18(5):408-412

7. Access Economics Pty Limited (2008) The burden of venous thromboembolism in Australia. Report for the Australian and New Zealand Working Party on the Management and Prevention of Venous Thromboembolism. http://www.accesseconomics. com.au/publicationsreports/getreport.php?report=161\&id=209. Accessed 20 October 2010

8. National Institute of Clinical Studies (2005) The incidence and risk factors for venous thromboembolism in hospitals in Western Australia 1999-2001. Prepared by The School of Population Health, University of Western Australia. NICS, Melbourne

9. Cohen AT, Tapson VF, Bergmann JF, Goldhaber SZ, Kakkar AK, Deslandes B, Huang W, Zayaruzny M, Emery L, Anderson FA Jr (2008) Venous thromboembolism risk and prophylaxis in the acute hospital care setting (ENDORSE study): a multinational cross-sectional study. Lancet 371(9610):387-394

10. University of Western Australia SoPH (2005) The Western Australian Venous Thromboembolism Study. Incidence and risk factors for venous thromboembolism in Western Australia 1999-2001. National Institute of Clinical Studies, Melbourne

11. Sandler DA, Martin JF (1989) Autopsy proven pulmonary embolism in hospital patients: Are we detecting enough deep vein thrombosis? J R Soc Med 82(4):203-205

12. Lindblad B, Sternby NH, Bergqvist D (1991) Incidence of venous thromboembolism verified by necropsy over 30 years. Br Med J 302(6778):709-711

13. Goldhaber SZ (2007) Venous thromboembolism risk among hospitalized patients: magnitude of the risk is staggering. Am J Hematol 82(9):775-776

14. Baglin T (2008) Venous thromboembolism in hospitalised patients: a public health crisis? Br J Haematol 141(6):764-770

15. Bergmann JF, Cohen AT, Tapson VF, Goldhaber SZ, Kakkar AK, Deslandes B, Huang B, Anderson FA Jr (2010) Venous thromboembolism risk and prophylaxis in hospitalised medically ill patients. The ENDORSE Global Survey. Thromb Haemost 103(4):736-748

16. Ageno W, Squizzato A, Ambrosini F, Dentali F, Marchesi C, Mera V, Steidl L, Venco A (2002) Thrombosis prophylaxis in medical patients: a retrospective review of clinical practice patterns. Haematologica 87(7):746-750

17. Ahmad HA, Geissler A, MacLellan DG (2002) Deep venous thrombosis prophylaxis: Are guidelines being followed? ANZ J Surg 72(5):331-334 
18. Arnold DM, Kahn SR, Shrier I (2001) Missed opportunities for prevention of venous thromboembolism: an evaluation of the use of thromboprophylaxis guidelines. Chest 120(6):1964-1971

19. Bratzler DW, Raskob GE, Murray CK, Bumpus LJ, Piatt DS (1998) Underuse of venous thromboembolism prophylaxis for general surgery patients: physician practices in the community hospital setting. Arch Intern Med 158(17):1909-1912

20. Burns PJ, Wilsom RG, Cunningham C (2001) Venous thromboembolism prophylaxis used by consultant general surgeons in Scotland. J R Coll Surg Edinb 46(6):329-333

21. Tooher R, Middleton P, Pham C, Fitridge R, Rowe S, Babidge W, Maddern G (2005) A systematic review of strategies to improve prophylaxis for venous thromboembolism in hospitals. Ann Surg 241(3):397-415

22. Declaration of Helsinki (2007) Ethical principles for medical research involving human subjects. Adopted 1964, last amended 2004: World Medical Association 2004. http://www.wma.net/e/ policy/b3.htm. Accessed 30 Sep 2007

23. Tapson VF, Decousus H, Pini M, Chong BH, Froehlich JB, Monreal M, Spyropoulos AC, Merli GJ, Zotz RB, Bergmann JF, Pavanello R, Turpie AG, Nakamura M, Piovella F, Kakkar AK, Spencer FA, Fitzgerald G, Anderson FA Jr (2007) Venous thromboembolism prophylaxis in acutely ill hospitalized medical patients: findings from the International Medical Prevention Registry on Venous Thromboembolism. Chest 132(3):936-945

24. Otero R, Uresandi F, Cayuela A, Blanquer J, Cabezudo MA, De Gregorio MA, Lobo JL, Nauffal D, Oribe M (2001) Use of venous thromboembolism prophylaxis for surgical patients: a multicentre analysis of practice in Spain. Eur J Surg 167(3): $163-167$
25. Amin A, Stemkowski S, Lin J, Yang G (2007) Thromboprophylaxis rates in US medical centers: success or failure? J Thromb Haemost 5(8):1610-1616

26. Eikelboom JW, Mazzarol A, Quinlan DJ, Beaver R, Williamson J, Yi Q, Hankey GJ (2004) Thromboprophylaxis practice patterns in two Western Australian teaching hospitals. Haematologica 89(5):586-593

27. Kucher N, Koo S, Quiroz R, Cooper JM, Paterno MD, Soukonnikov B, Goldhaber SZ (2005) Electronic alerts to prevent venous thromboembolism among hospitalized patients. N Engl J Med 352(10):969-977

28. Durieux P, Nizard R, Ravaud P, Mounier N, Lepage E (2000) A clinical decision support system for prevention of venous thromboembolism: effect on physician behavior. JAMA 283(21): 2816-2821

29. Campbell PJ, Arbuthnot C, Reed M, Passlow C, Vagg M, Orford N, Tomlinson S (2009) PreVent—an electronic risk assessment tool and decision support in acute inpatients. Thromb Haemost 7(Suppl 2):270-271

30. Grol R, Grimshaw J (2003) From best evidence to best practice: effective implementation of change in patients' care. Lancet 362(9391):1225-1230

31. Maynard G, Stein J (2010) Designing and implementing effective venous thromboembolism prevention protocols: lessons from collaborative efforts. J Thromb Thrombolysis 29(2):159-166

32. Grimshaw JM, Eccles MP, Walker AE, Thomas RE (2002) Changing physicians' behavior: what works and thoughts on getting more things to work. J Contin Educ Health Prof 22(4): $237-243$ 